\title{
Location of Pc 1-2 waves relative to the magnetopause
}

\author{
R. E. Denton, J. LaBelle, and X. Zhu \\ Department of Physics and Astronomy, Dartmouth College, Hanover, NH 03755, USA
}

Received: 21 May 2002 - Revised: 15 July 2002 - Accepted: 17 July 2002

\begin{abstract}
Spacecraft-borne and ground-based magnetometers frequently detect magnetospheric micropulsations in the period range $0.2-10 \mathrm{~s}$, termed Pc $1-2$, and attributed to electromagnetic ion cyclotron waves driven by temperature anisotropy $\left(T_{\perp}>T_{\|}\right)$. Previous surveys of Pc 1 occurrence locations have been limited to $L \leq 9$. We present AMPTE/IRM observations of the distribution of Pc 1 waves out to the magnetopause, for a limited region of MLT = 10-14. The probability of wave occurrence $P_{\text {wav }}$ is large (>0.15) between $L=7-12$, peaking at $L=8-10\left(P_{\text {wav }} \sim\right.$ $0.25)$. When the $\mathrm{L}$-value is normalized to the magnetopause position $L_{\mathrm{mp}}$, however, the highest probabilities of Pc 1 wave occurrence are close to the magnetopause, with $P_{\text {wav }} \sim 0.25$ for $L_{\text {norm }} \equiv L / L_{\mathrm{mp}}=0.8-1.0$. These results are consistent with increased convective growth rate at large $L$ and with the greater effect of magnetosphere compression close to the magnetopause. On the other hand, we only directly observe magnetic field compression for at most about $25 \%$ of the wave events.
\end{abstract}

Key words. Magnetospheric physics (magnetospheric configuration and dynamics; MHD waves and instabilities; plasma waves and instabilities)

\section{Introduction}

Magnetospheric micropulsations in the period range 0.2$10 \mathrm{~s}$, termed Pc 1-2, are commonly observed on the ground and in space, and are thought to be associated with electromagnetic ion cyclotron (EMIC) waves driven by temperature anisotropy $\left(T_{\perp}>T_{\|}\right)$(Anderson et al., 1996). As discussed by Anderson et al. (1992a) and Fraser and Nguyen (2001), early theoretical considerations favored the region just inside the plasmapause as the most likely location for the production of EMIC waves. This outer region of the plasmasphere would overlap with the ring current population (which

Correspondence to: R. E. Denton

(richard.denton@dartmouth.edu) drives the instability), yet would have small group velocity $v_{g} \sim V_{A} \sim \rho^{-1 / 2}$, where $V_{A}$ is the Alfvén speed and $\rho$ is the mass density. Thus, the convective growth rate $S \sim \gamma / v_{g}$ would be large, where $\gamma$ is the temporal growth rate.

Nevertheless, some early models (Criswell, 1969; Perraut et al., 1976) indicated that there could be significant wave amplification at $L>7$, depending on the plasma model. Indeed, spacecraft observations show that EMIC waves are prevalent in the outer magnetosphere $L>7$ (Erlandson et al., 1990), consistent with the results of some earlier groundbased data (Fraser, 1968). Anderson et al. (1992a, b) provide the most extensive statistical survey of Pc 1 wave properties using spacecraft data, showing that the occurrence frequency of Pc 1 waves peaks in the early afternoon and increases out to the outermost L-shell sampled by AMPTE/CCE $\sim 9$. They explain this increase with a simple magnetospheric model in which the decrease in magnetic field $B$ (dipole model) at large $L$ contributes significantly to a decrease in $v_{g} \sim V_{A} \sim B$. Fraser and Nguyen (2001) studied Pc 1 spatial occurrance using the CRRES satellite, which includes electron density measurements, allowing the plasmapause position to be identified. They found a slight enhancement of Pc 1 wave power at the plasmapause, but concluded that Pc 1 occurrence does not maximize at the plasmapause; instead it predominates in the afternoon and increases with radial distance, consistent with the results of Anderson et al. (1992a).

Olson and Lee (1983) reviewed ground magnetometer observations which connect the occurrence of Pc 1 waves with sudden compressions of the magnetosphere (or sudden impulse SI). From double adiabatic theory, they show that compressions lead to an increase in the hot particle anisotropy $A \equiv\left(T_{\perp}-T_{\|}\right) / T_{\|}-1$, which in turn leads to the stimulation of Pc 1 waves. In fact, Anderson et al. (1996) showed that EMIC instability can be roughly predicted if $A \beta_{\|}^{1 / 2} \gtrsim 1$, where $\beta_{\|}$is the ratio of the parallel plasma pressure to the magnetic pressure. The model of Olson and Lee (1983) shows that compressions do not significantly change $\beta_{\|}$, but can lead to a significant increase in $A$. Erlandson et al. (1994) 


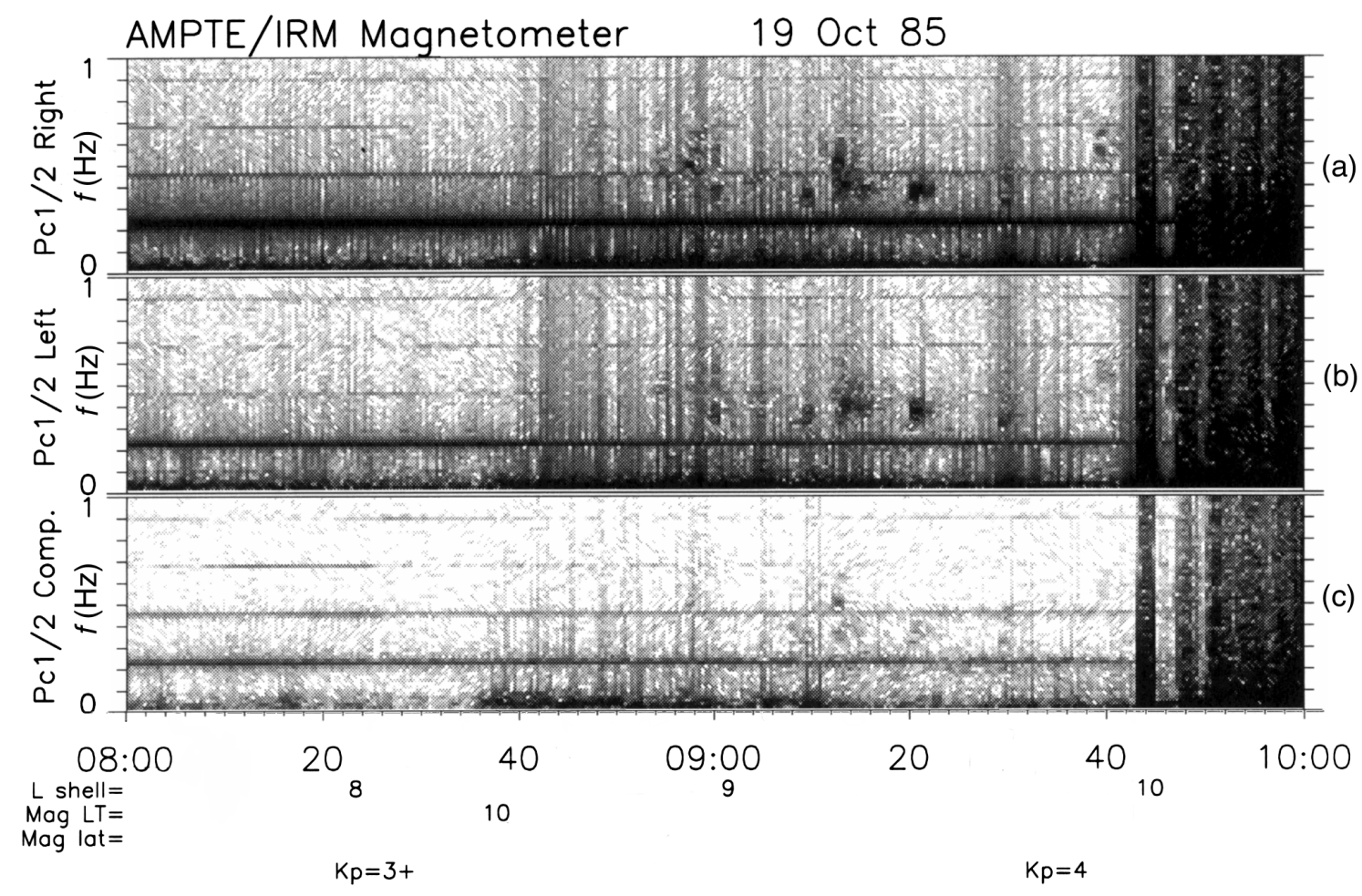

Fig. 1. Survey spectrogram of right-hand polarized (top panel), left-hand polarized (middle panel), and compressional (bottom panel) Pc 1 waves detected by the IRM flux-gate magnetometer on 19 October 1985. In this example, Pc 1 waves occur starting at 08:54 UT and continue to be detected intermittently until the IRM passes into the magnetosheath at about 09:48 UT. The strong line at $0.22 \mathrm{~Hz}$ is due to satellite spin and should be ignored.

present an example of a compression-induced Pc 1 event associated with a magnetospheric substorm in which the Pc 1 waves turn on and off with the precise timing of the compression. Anderson and Hamilton (1993) show that the probability of observing Pc 1 waves in space is significantly enhanced during magnetospheric compressions. In their view, the magnetosphere is usually near marginal stability to EMIC waves, and magnetospheric compressions lead to increased anisotropy $A$ and readily switch the magnetosphere to the unstable state. This model explains the higher occurrence rates of Pc1 waves at large $L$ by Anderson et al. (1992a) (up to the maximum L-value sampled by AMPTE/CCE), because compressions have a larger effect on the region close to the magnetopause than on the inner magnetosphere.

Engebretson et al. (2002) examined the set of Pc 1 events observed simultaneously by an array of ground stations in Antarctica and by the Polar spacecraft during the first 18 months of satellite operation. They found that $75 \%$ of these events occurred at the same time as magnetospheric compressions, although about half of these events are associated with spatially localized distribution functions (apparently another cause of EMIC waves). Several of their spatially localized events are not correlated with magnetospheric compressions. They showed evidence that these distribution functions may result from convergence of drift paths from injection on the nightside or stagnation of flow along certain drift paths. Furthermore, some Pc 1 events, though perhaps only a small percentage, are directly associated with particle interactions associated with substorms (Erlandson and Ukhorskiy, 2001).

Anderson et al. (1992a) and Fraser and Nguyen (2001) indicate that Pc 1 waves are more likely at large radii but do not determine the distribution of waves at very large $L$ due to the orbits of the spacecraft used (apogee of $R \sim 9 R_{\mathrm{E}}$ for AMPTE/CCE and $\sim 7 R_{\mathrm{E}}$ for CRRES). In this paper, we use magnetometer data from the AMPTE/IRM spacecraft $\left(19 R_{\mathrm{E}}\right.$ apogee) to examine the L-shell distribution of Pc 1 waves in the magnetic local time (MLT) range 10-14.

\section{Data analysis}

The AMPTE/IRM spacecraft, operated between August 1984 and August 1986, had an apogee of $\approx 19 R_{\mathrm{E}}$ and an inclination $\approx 27 \mathrm{deg}$. It included plasma, magnetic field, energetic particle, and wave experiments. The data used in this study are from the three-axis flux-gate magnetometer which measured the vector magnetic field 32 times per second (Lühr et al., 1985). Since the full vector is measured, the magnetic field can be broken into right-hand, left-hand, and compressional components by transforming to mean field coordinates (with $z$ along the mean field), Fouriertransforming, and recombining the complex Fourier transforms of the $x$ and $y$ components into $B_{L, R}=B_{x} \pm i B_{y}$ 
before computing the power spectrum. This procedure is outlined by Kodera et al. (1977) and used in many subsequent papers (e.g. LaBelle and Treumann, 1992, who apply the technique to AMPTE/IRM data).

Figure 1 shows example Pc 1 wave survey spectrograms produced from the IRM vector magnetometer data from 08:00-10:00 UT on 19 October 1985, when IRM was located at approximately 10:00 MLT and moving outward, intersecting field lines corresponding to L-shells ranging from 7.4 to $10.3 \mathrm{R}_{\mathrm{E}}$. The $\mathrm{L}$-shell values are determined by mapping the field line of the spacecraft, assuming the dipole field model, and computing its equatorial stand-off distance. In Fig. 1, panels a-c represent right-polarized, left-polarized, and compressional waves, respectively. The horizontal line at $0.22 \mathrm{~Hz}$ (and harmonics) results from the spacecraft spin and should be ignored. On 19 October, the spacecraft encounters the magnetopause at 09:44-09:48 UT ( $L \approx 10.2 R_{\mathrm{E}}$ ), and after that time broadband, large amplitude fluctuations, characteristic of the magnetosheath, dominate all three components of magnetic field. Inside the magnetosphere, Pc 1 waves appear as a series of pearl-like emissions on frequencies 0.25 $0.60 \mathrm{~Hz}$, starting at 08:54 UT $\left(L=8.8 R_{\mathrm{E}}\right)$ and continuing intermittently right up to the magnetopause. Such events are easily identified in the Pc 1 wave survey spectrograms archived at Dartmouth.

For a statistical study, we examined survey spectrograms similar to Fig. 1 for all times when AMPTE/IRM was within the magnetosphere for MLT in the range 10:00-14:00, and for which the satellite collected data during the nearest magnetopause crossing either immediately before or immediately after, providing an estimate of the magnetopause position. For each ten minutes of data, we noted the L-value, the number of minutes of wave data (excluding data gaps), and the number of minutes during which Pc 1 waves $(0.1-$ $1 \mathrm{~Hz}$ ) were observed above the noise level of the survey plots. Each survey plot is independently scaled to optimize detection of events, but power spectral densities as small as 0.5$1.0 \mathrm{nT}^{2} / \mathrm{Hz}$ are easily detectable (see, for example, Fig. 1 of LaBelle and Treumann, 1992). Altogether, there were 47 IRM orbits which met our criteria, and Pc 1 waves were observed for at least for some range of $L$ during 37 of these.

The upper limit of frequency sampled in our study is $1 \mathrm{~Hz}$. The lower limit is harder to define, but is approximately $0.1 \mathrm{~Hz}$. The question arises as to whether or not we are able to detect all EMIC waves occuring at a particular time. Based on the distribution of frequencies in Fig. 10 of Anderson et al. (1992a), and assuming a frequency bandwidth of $\pm 30 \%$ typical of events in their study, our $1 \mathrm{~Hz}$ upper limit causes us to miss about $1 \%$ of the EMIC events for $L=5-6$, and less for higher L-values. For $L=4-5$, however, we detect only about half of EMIC events. This problem is accentuated for the MLT range 10-14, since the highest frequencies occur in that range (Fig. 9 of Anderson et al. (1992a)), yet even in that range, the vast majority of events occurring at $L \geq 6$ have a frequency $<1 \mathrm{~Hz}$. Therefore, the $1-\mathrm{Hz}$ limit is not a problem for $L \geq 6$, but our study significantly underestimates the occurence probabilities for $L<5$. As seen from Fig. 9 of

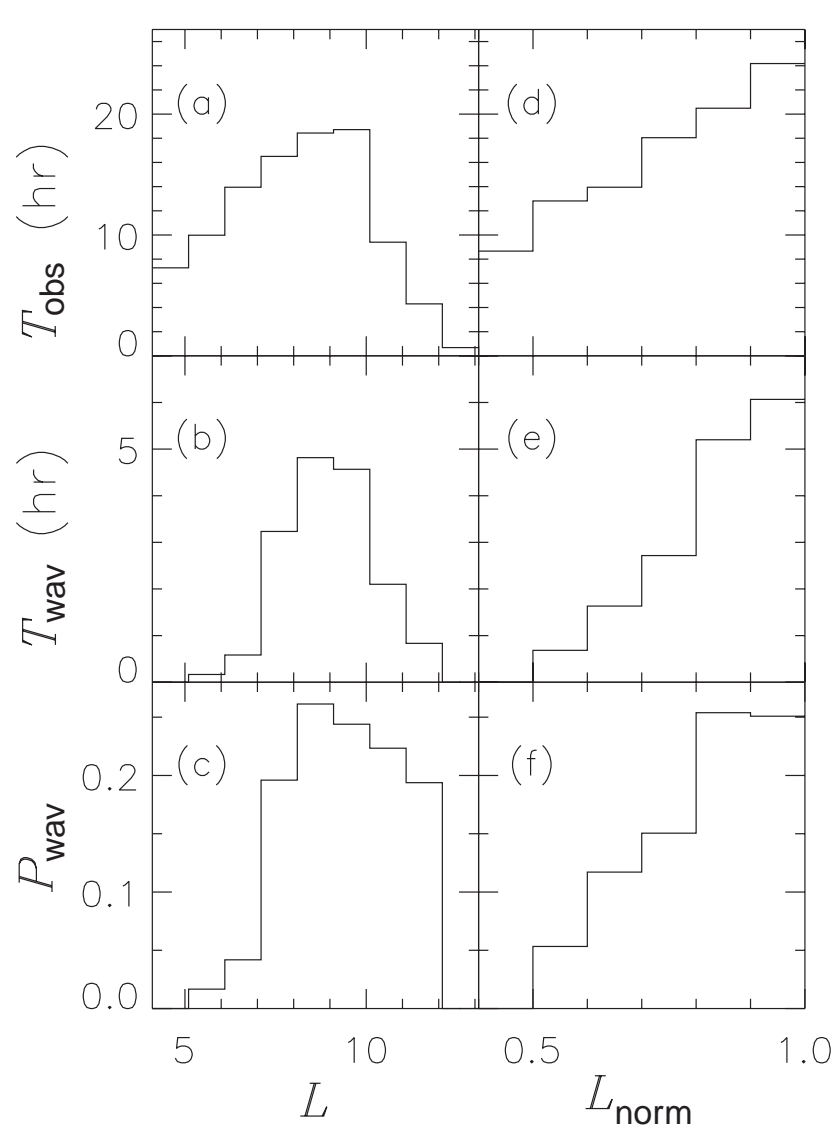

Fig. 2. (a) Total time of observations $T_{\text {obs }}$ in hours, (b) total time of Pc 1 wave activity $T_{\text {wav }}$ in hours, and (c) Pc 1 wave occurrence probability $P_{\mathrm{wav}}=T_{\mathrm{wav}} / T_{\mathrm{obs}}$ in $L$ bins $3.1-4.1,4.1-5.1$, ..., 12.1-13.1.

Panels (d), (e), and (f) show the same quantities as were plotted in (a), (b), and (c), respectively, but now plotted versus $L_{\text {norm }} \equiv$ $L / L_{\mathrm{mp}}$ for $L_{\text {norm }}$ bins $0.4-0.5,0.5-0.6, \ldots, 0.9-1.0$.

Anderson et al. (1992a), the $0.1 \mathrm{~Hz}$ lower limit should not be a problem for MLT $=10-14$.

Figure 2 shows the results of this statistical study. In Figs. $2 \mathrm{a}-\mathrm{c}$, we show the total time of observations $T_{\mathrm{obs}}$, the total time of Pc 1 wave activity $T_{\mathrm{wav}}$, and the wave occurrence probability $P_{\text {wav }}=T_{\text {wav }} / T_{\text {obs }}$, respectively, for $L$ bins 3.1-4.1, 4.1-5.1, .., 12.1-13.1. As discussed above, the probability of EMIC wave occurrence at $L=4-5$ is not reliable, and the probability at $L=5-6$ may be slightly higher than we measure. Nevertheless, Fig. $2 c$ clearly shows that the occurrence probability drops steeply at low $L$, as evidenced by the values of $P_{\text {wav }}$ at $L=5-6$ and 6-7. The actual occurrence rate for $L=4-5$ is probably very small, though it may not be as low as zero, as was found in this study restricted to $f \leq 1 \mathrm{~Hz}$.

The uncertainties in $P_{\text {wav }}$ are difficult to estimate. Modelling after a Gaussian distribution, a rough estimate can be found by dividing the $P_{\text {wav }}$ values by the square root of the number of orbits (statistically distinct events). However, the data for a particular orbit did not always span all the $L$ bins. 
Typically, there were data from about 30 orbits averaged in each bin. Thus, we estimate a $1 / \sqrt{30}=18 \%$ uncertainty.

The values of $P_{\text {wav }}$ shown in Fig. 2c are consistent with the results of Anderson et al. (1992a) up to the maximum L-value sampled in their survey $\sim 9$. Figure $2 \mathrm{c}$ shows that $P_{\text {wav }}$ reaches its highest value at $L=8-10$, and that it decreases for larger $L$. Such a result does not necessarily indicate that the wave probability decreases as the magnetopause is approached. The position of the magnetopause is not the same for all the data plotted in Fig. 2c. In fact, the nominal magnetopause location is roughly at $L=10$, and the largest L-values in Fig. 2c (12.1-13.1) will only be within the magnetosphere (inside the magnetopause) when the solar wind pressure is low and the magnetopause is relatively uncompressed. Under such conditions, the Pc 1 wave occurrence probability might be low, as discussed in Sect. 1 .

In order to determine the distribution of Pc 1 wave probability with respect to the magnetopause location, Figs. 2d-e show the same quantities as were shown in Figs. 2a-c, but plotted versus $L_{\text {norm }} \equiv L / L_{\mathrm{mp}}$, where $L_{\mathrm{mp}}$ is the L-value of the magnetopause for the particular orbital pass. In some cases, there were a number of closely spaced magnetopause crossings and we chose the average position among these crossings. In most cases, the difference in the L-value between the closest value (smallest $L$ ) and this "average" value was small ( $\left.\sim 0.1 \mathrm{R}_{\mathrm{E}}\right)$, although it sometimes ranged to be as much as $0.5 R_{\mathrm{E}}$. Figure $2 \mathrm{f}$ shows that for the probability of Pc 1-2 wave occurrence $P_{\text {wav }}$ increases for locations near the magnetopause. The value of $P_{\mathrm{wav}}$ is the same for the $0.8-0.9$ and $0.9-1.0$ bins within the statistical uncertainty.

Finally, we examined the magnitude of the magnetic field observed during the Pc 1 events of this survey in an effort to determine whether they are associated with compressions of the magnetosphere. At most, about $25 \%$ of the observed Pc1 events were associated with enhancements (of at least $\sim 15 \%$ ) in the magnetic field, which may indicate compressions. Anderson and Hamilton (1993) observed that when the AMPTE/CCE data are screened to include only times of magnetospheric compressions, the Pc 1 occurrence rate was enriched from the normal value $\sim 20-25 \%$ to $63 \%$. Our data would be consistent with those of Anderson and Hamilton if such compressions occur about $10 \%$ of the time. On the other hand, our fraction of compression related events seems to be significantly less than that found by Engebretson et al. (2002).

\section{Summary}

The most comprehensive survey of $\mathrm{Pc} 1$ occurrence locations to date is that of Anderson et al. (1992a), but their survey is limited to $L \leq 9$. Using AMPTE/IRM data, we measure the distribution of Pc 1 waves out to larger $L$ for a limited region of MLT $=10-14$. We find that the probability of wave occurrence $P_{\text {wav }}$ is large ( $>0.15$ ) between $L=7-12$, peaking at $L=8-10\left(P_{\text {wav }} \sim 0.25\right)$. When the $\mathrm{L}$-value is normalized to the magnetopause position $L_{\mathrm{mp}}$, the highest probabilities of Pc 1 wave occurrence are close to the magnetopause, with $P_{\text {wav }} \sim 0.25$ for $L_{\text {norm }} \equiv L / L_{\mathrm{mp}}=0.8-1.0$. These results are consistent with increased convective growth rate at large $L$ (Anderson et al., 1992a), and with the greater effect of magnetosphere compression close to the magnetopause (Anderson and Hamilton, 1993). On the other hand, we only directly observe magnetic field compression for at most about $25 \%$ of the wave events.

Acknowledgements. We acknowledge the help of Thaddeus Law with this research. Work at Dartmouth was supported by NASA grant NAG5-11712.

Topical Editor G. Chanteur thanks A. Potapor and another referee for their help in evaluating this paper.

\section{References}

Anderson, B. J. and Hamilton, D. C.: Electromagnetic ion cyclotron waves stimulated by modest magnetospheric compressions, J. Geophys. Res., 98, 11369-11 382, 1993.

Anderson, B. J., Erlandson, R. E., and Zanetti, L. J.: A statistical study of Pc 1-2 magnetic pulsations in the equatorial magnetosphere, 1, Equatorial occurrence distributions, J. Geophys. Res., 97, 3075-3088, 1992a.

Anderson, B. J., Erlandson, R. E., and Zanetti, L. J.: A statistical study of Pc 1-2 magnetic pulsations in the equatorial magnetosphere, 2, Wave properties, J. Geophys. Res., 97, 3089-3101, 1992 b.

Anderson, B. J., Denton, R. E., Ho, G., Hamilton, D. C., Fuselier, S. A., and Strangeway, R. J.: Observational test of local proton cyclotron instability in the Earth's magnetosphere, J. Geophys. Res., 101, 21 527-21 544, 1996.

Criswell, D. R.: Pc 1 micropulsation activity and magnetospheric amplification of 0.2- to 5.0-Hz hydrodynamic waves, J. Geophys. Res., 74, p. 205, 1969.

Engebretson, M. J., Peterson, W. K., Posch, J. L., Klatt, M. R., Anderson, B. J., Russell, C. T., Singer, H. J., Arnoldy, R. L., and Fukunishi, H.: Observations of two types of Pc 1-2 pulsations in the outer dayside magnetosphere, J. Geophys. Res., in press, 2002.

Erlandson, R. E. and Ukhorskiy, A. J.: Observations of electromagnetic ion cyclotron waves during geomagnetic storms: Wave occurrence and pitch angle scattering, J. Geophys. Res., 106, 38833896, 2001.

Erlandson, R. E., Zanetti, L. J., Potemra, T. A., Block, L. P., and Holmgren, G.: Viking magnetic and electric field observations of Pc 1 waves at high latitudes, J. Geophys. Res., 95, 5941-5955, 1990.

Erlandson, R. E., Zanetti, L. J., Engebretson, M. J., Arnoldy, R. L., Bösinger, T., and Mursula, K.: Pc 1 waves generated by a magnetospheric compression during the recovery phase of a geomagnetic storm, in: Solar Wind Sources of Magnetospheric UltraLow-Frequency Waves, (Eds) Engebretson, M. J., Takahashi, K., and Scholer, M., AGU, Washington D. C., 1994.

Fraser, B. J.: Temporal variations in Pc 1 geomagnetic micropulsations, Planet. Space Sci., 16, p111, 1968.

Fraser, B. J. and Nguyen, T. S.: Is the plasmapause a preferred source region of electromagnetic ion cyclotron waves in the magnetosphere?, J. Atmos. Solar-Terr. Phys., 63, 1225-1247, 2001. 
Kodera, K., Gendrin, R., and De Villedary, C.: Complex representation of a polarized signal and its application to ULF waves, J. Geophys. Res., 82, 1245-1255, 1977.

LaBelle, J. and Treumann, R. A.: Poynting vector measurements of electromagnetic ion cyclotron waves in the plasmasphere, J. Geophys. Res., 97, 13 789-13 797, 1992.

Lühr, H., Klöcker, N., Oelschlägel, W., Häusler, B., and Acuña, M.:
The IRM fluxgate magnetometer, IEEE Trans. Geosci. Remote Sens., GE-23, p. 259, 1985.

Olson, J. V. and Lee, L. C.: Pc 1 wave generation by sudden impulses, Planet. Space Sci., 31, p. 295, 1983.

Perraut, S., Gendrin, R., and Roux, A.: Amplification of ioncyclotron waves for various typical radial profiles of magnetospheric parameters, Atmos. Terr. Phys., 38, p. 1191, 1976. 